\title{
THE NONEXISTENCE OF CERTAIN TIGHT SPHERICAL DESIGNS
}

\author{
E. BANNAI, A. MUNEMASA, AND B. VENKOV
}

\begin{abstract}
In this paper, the nonexistence of tight spherical designs is shown in some cases left open to date. Tight spherical 5-designs may exist in dimension $n=$ $(2 m+1)^{2}-2$, and the existence is known only for $m=1,2$. In the paper, the existence is ruled out under a certain arithmetic condition on the integer $m$, satisfied by infinitely many values of $m$, including $m=4$. Also, nonexistence is shown for $m=3$. Tight spherical 7-designs may exist in dimension $n=3 d^{2}-4$, and the existence is known only for $d=2,3$. In the paper, the existence is ruled out under a certain arithmetic condition on $d$, satisfied by infinitely many values of $d$, including $d=4$. Also, nonexistence is shown for $d=5$. The fact that the arithmetic conditions on $m$ for 5-designs and on $d$ for 7-designs are satisfied by infinitely many values of $m$ and $d$, respectively, is shown in the Appendix written by Y.-F. S. Pétermann.
\end{abstract}

\section{§1. INTRODUCTION}

The concept of a spherical $t$-design is due to Delsarte-Goethals-Seidel [7]. For a positive integer $t$, a finite nonempty set $X$ in the unit sphere

$$
S^{n-1}=\left\{x=\left(x_{1}, x_{2}, \ldots, x_{n}\right) \in \mathbb{R}^{n} \mid x_{1}^{2}+x_{2}^{2}+\cdots+x_{n}^{2}=1\right\}
$$

is called a spherical t-design in $S^{n-1}$ if the following condition is satisfied:

$$
\frac{1}{|X|} \sum_{x \in X} f(x)=\frac{1}{\omega_{n-1}} \int_{S^{n-1}} f(x) d S
$$

for all polynomials $f(x)=f\left(x_{1}, x_{2}, \ldots, x_{n}\right)$ of degree not exceeding $t$. Here, the righthand side involves integration on the sphere, and $\omega_{n-1}$ denotes the volume of the sphere $S^{n-1}$. The meaning of the notion of a spherical $t$-design is that it is a finite set of points on the sphere that replaces the sphere itself with respect to the integration of any polynomial of degree up to $t$. So, it is a finite set of points on the sphere that "approximates" the sphere. It is known [7] that there is a lower bound (Fisher type inequality) for the size of a spherical $t$-design in $S^{n-1}$. Namely, if $X$ is a spherical $t$-design in $S^{n-1}$, then

$$
|X| \geq\left(\begin{array}{c}
n-1+[t / 2] \\
{[t / 2]}
\end{array}\right)+\left(\begin{array}{c}
n+[t / 2]-2 \\
{[t / 2]-1}
\end{array}\right)
$$

if $t$ is even, and

$$
X \geq 2\left(\begin{array}{c}
n-1+[t / 2] \\
{[t / 2]}
\end{array}\right)
$$

if $t$ is odd. From the design-theoretical viewpoint, for $t$ and $n$ given, to find a $t$-design $X$ with the cardinality $|X|$ as small as possible is the most interesting problem. A

2000 Mathematics Subject Classification. Primary 05B30.

The third author was partially supported by the Swiss National Science Foundation. This work was done during a three month visit of B. Venkov to Kyushu University, and he thanks the University for hospitality.

The paper contains Appendix written by Y.-F. S. Pétermann. 
$t$-design $X$ for which one of the lower bounds mentioned above is attained, is called a tight $t$-design, and such a design enjoys many interesting properties ([1], [6]-8], etc.). Unfortunately, tight $t$-designs rarely exist, and in [2, 3] it was proved that if a tight $t$-design in $S^{n-1}$ with $n \geq 3$ exists, then necessarily either $t \leq 5$, or $t=7,11$.

The tight $t$-designs with $t=1,2,3$ as well as $t=11$ are classified completely, while classification of tight $t$-designs for $t=4,5,7$ is still an open problem. It is known that the existence of a tight 4-design in $S^{n-2}$ is equivalent to the existence of a tight 5-design in $S^{n-1}$. It is also known that if a tight 5-design $X$ exists in $S^{n-1}(n \geq 3)$, then either $n=3$ and $X$ coincides with the 12 vertices of the icosahedron, or $n=(2 m+1)^{2}-2$ for an integer $m$ (see [1]-3] and [7]). This statement comes from the fact that all the eigenvalues of the corresponding association scheme are rational (see [2, Theorem 2]).

Note that, as was mentioned in [1. Lemma 8.3.7, p. 175], the case of $t=5$ and $n=3$ (i.e., the case of the 12 vertices of the icosahedron) was omitted in the original statement of Theorem 2 in [2], and this omission is the only one. So, Theorem 2 in [2] remains true with this minor modification. Existence is known only for $m=1,2$, and such designs are known to be unique [9].

If a tight 7-design exists in $S^{n-1}(n \geq 3)$, then $n=3 d^{2}-4$ (see [1]-3] and [7]). Existence is known only for $d=2,3$, and the corresponding designs are known to be unique [4].

In this paper, we study the existence of tight 5-designs (hence, of tight 4-designs as well) and tight 7-designs, and prove the following results. No tight 5-designs in $S^{(2 m+1)^{2}-2}$ exist for the first two open cases where $m=3,4(n=47,79)$ as well as for certain values of $m$ (see Theorem 3.1 for the exact statement). No tight 7-designs in

$S^{3 d^{2}-4}$ exist for the first two open cases where $d=4,5(n=44,71)$ as well as for certain values of $d$ (see Theorem 4.1 for the exact statement).

The proof of nonexistence is based on the study of the Euclidean lattice generated by our design $X$, normalized so as to make all scalar products integer-valued. Using the basic relations (1)-(3), we obtain enough information on the determinant of this lattice. For the dimensions $n=44$ and 71, the lattice is unimodular, and for the dimension $n=47$, a closely related lattice $\Gamma$ (see Subsection [3.2) happens to be even and of determinant 2 or 6 . We exclude the case of determinant 6 by the Milgram-Braun formula. If $\operatorname{det} \Gamma=2$, then we glue $\Gamma$ with an $A_{1}$-lattice $\sqrt{2} \mathbb{Z}$ to obtain an even unimodular lattice of dimension 48. Calculating the theta series of the last-mentioned lattice in two ways (from the design and by modular forms), we arrive at a contradiction.

We note that our method is essentially the same as that used in [11] by Martinet [11] whose result leads to the nonexistence proof in the case of $n=71$ (see Theorem 4.2).

We have not succeeded in proving the nonexistence of a tight 7-design in dimension $n=104$, so that this case remains to be an interesting problem. We give some results concerning this case at the end of the paper.

\section{§2. Preliminaries}

Let $S^{n-1}(d)$ denote the $(n-1)$-dimensional sphere in $\mathbb{R}^{n}$ of radius $\sqrt{d}$ and centered at the origin. A finite subset $D$ of $S^{n-1}(d)$ is called a spherical t-design if $\frac{1}{\sqrt{d}} D$ is a spherical $t$-design in the unit sphere.

It is more convenient to consider spherical designs in a sphere of an appropriate radius than those in the unit sphere, because then we can make all the scalar products integervalued in some cases. 
Let $D$ be a finite subset of $S^{n-1}(d)$ satisfying $D=-D$. Then we may write $D=$ $X \cup-X$ with $X \cap-X=\varnothing$. If $D$ is a spherical 5-design, then for all $\alpha \in \mathbb{R}^{n}$ we have

$$
\begin{aligned}
\sum_{x \in X}(\alpha, x)^{2} & =\frac{d}{n}|X|(\alpha, \alpha), \\
\sum_{x \in X}(\alpha, x)^{4} & =\frac{3 d^{2}}{n(n+2)}|X|(\alpha, \alpha)^{2} .
\end{aligned}
$$

If, moreover, $D$ is a spherical 7 -design, then

$$
\sum_{x \in X}(\alpha, x)^{6}=\frac{15 d^{3}}{n(n+2)(n+4)}|X|(\alpha, \alpha)^{3} .
$$

These three identities are called the basic relations. For the proof of these, we refer the reader to [16. We mention a consequence of these relations, which we shall need later:

$$
\sum_{x \in X}(x, \alpha)\left((x, \alpha)^{2}-1\right)\left((x, \alpha)^{2}-4\right) x=(\text { scalar }) \alpha .
$$

Suppose that the scalar products of arbitrary elements of $X$ are integers. Let $\Lambda$ be the lattice generated by $X$. For $\alpha \in \Lambda^{*}$, we define

$$
n_{k}(\alpha)=|\{x \in X \mid(\alpha, x)= \pm k\}| \quad(k=0,1,2, \ldots) .
$$

Then, obviously,

$$
\sum_{k=0}^{\infty} n_{k}(\alpha)=|X|
$$

Now the basic relations (1)-(3) can be written as

$$
\begin{aligned}
& \sum_{k=1}^{\infty} k^{2} n_{k}(\alpha)=\frac{d}{n}|X|(\alpha, \alpha), \\
& \sum_{k=1}^{\infty} k^{4} n_{k}(\alpha)=\frac{3 d^{2}}{n(n+2)}|X|(\alpha, \alpha)^{2}, \\
& \sum_{k=1}^{\infty} k^{6} n_{k}(\alpha)=\frac{15 d^{3}}{n(n+2)(n+4)}|X|(\alpha, \alpha)^{3},
\end{aligned}
$$

respectively.

We shall also need the Milgram-Braun formula for an even integral lattice $\Gamma$ (see [12, 15]):

$$
\sum_{\alpha+\Gamma \in \Gamma^{*} / \Gamma} e^{\pi i(\alpha, \alpha)}=\sqrt{\operatorname{det} \Gamma} e^{\pi i \operatorname{dim} \Gamma / 4} .
$$

A consequence of this formula is that an even unimodular lattice exists only if the dimension is a multiple of 8 . This fact will be needed to rule out the existence of a tight 7-design in dimension 44 .

If an integral lattice $\Gamma$ is odd, then we denote by $\Gamma_{+}$the sublattice consisting of elements of even norm. For an odd unimodular integral lattice $\Gamma$, it is known that there exists an element $\alpha \in \Gamma$, unique up to modulo $2 \Gamma$, such that

$$
(\alpha, \alpha) \equiv \operatorname{dim} \Gamma(\bmod 8)
$$

and, for $x \in \Gamma$,

$$
(\alpha, x) \text { is even if and only if } x \in \Gamma_{+} .
$$


Such an element $\alpha$ is called a characteristic vector, and $\frac{1}{2} \alpha$ belongs to the shadow of $\Gamma$.

Let $p$ be a prime. The $p$-adic valuation $\nu_{p}(x)$ of a nonzero rational number $x$ is defined to be the integer $k$ such that $x=p^{k} a / b$, where $a, b$ are relatively prime integers and $(a, p)=(b, p)=1$.

\section{§3. Tight 5-DESigns}

3.1. Basic results. It is known that a tight 5-design $D$ in the unit sphere $S^{n-1}(1) \subset \mathbb{R}^{n}$ is of cardinality $n(n+1) / 2$ and is antipodal, i.e., $D=-D$. The values of the scalar products of elements of $D$ are

$$
A(D)=\{(x, y) \mid x, y \in X, x \neq y\}=\{ \pm \alpha,-1\}
$$

with $\alpha=1 / \sqrt{n+2}$ for $n>3$. First we note that if $D$ is a tight 5 -design in $S^{n-1}(1)$, then for an arbitrary fixed $x_{0} \in X$, the set

$$
D^{\prime}=\frac{1}{\sqrt{1-\alpha^{2}}} D_{0} \subset S^{n-2}(1),
$$

where

$$
D_{0}=\left\{x \in D \mid\left(x, x_{0}\right)=\alpha\right\},
$$

is a tight 4-design in $S^{n-2}(1)$. Conversely, a tight 4 -design in $S^{n-2}(1)$ yields a tight 5 -design in $S^{n-1}(1)$.

In Theorem 2 of 2] it was claimed that, for a tight $t$-design $D$ in $S^{n-1}(1)$, all the elements of $A(D)$ must be rational. An obvious exception exists for this claim, namely, the icosahedron in the case where $(t, n)=(5,3)$. As was shown in [1, Lemma 8.3.7], this case is the only exception, and the original statement of Theorem 2 in [2] becomes true with this modification. So, with the assumption that $n>3$, we conclude that $n+2$ is a square. Moreover, we can easily see that $n$ must be odd, since otherwise the parameters of the strongly regular graph associated with the tight 4-design become nonintegral.

Suppose that $D=X \cup-X \subset S^{n-1}(d) \subset \mathbb{R}^{n}$ is a tight spherical 5-design, where $X \cap-X=\varnothing$ and

$$
\begin{aligned}
& n=d^{2}-2 \quad(d \in \mathbb{N}), \\
& d=2 m+1 \quad(m \in \mathbb{N}) .
\end{aligned}
$$

Then

$$
\begin{aligned}
|X| & =\frac{n(n+1)}{2}, \\
(x, y) & = \pm 1 \quad \text { for all } x, y \in X, x \neq y .
\end{aligned}
$$

Now the basic relations (7) and (8) can be written as

$$
\begin{aligned}
& \sum_{k=1}^{\infty} k^{2} n_{k}(\alpha)=2 m(m+1)(2 m+1)(\alpha, \alpha), \\
& \sum_{k=1}^{\infty} k^{4} n_{k}(\alpha)=6 m(m+1)(\alpha, \alpha)^{2},
\end{aligned}
$$

respectively. We also mention another relation, which can easily be derived from (2):

$$
\begin{aligned}
\sum_{x \in X} & (\alpha, x)(\beta, x)(\gamma, x)(\delta, x) \\
& =2 m(m+1)((\alpha, \beta)(\gamma, \delta)+(\alpha, \gamma)(\beta, \delta)+(\alpha, \delta)(\beta, \gamma)),
\end{aligned}
$$

where $\alpha, \beta, \gamma, \delta \in \mathbb{R}^{n}$. 
Let $\Lambda$ be the lattice generated by $X$. Since $(x, y)$ is odd for all $x, y \in X$, we see that the even sublattice $\Lambda_{+}$of $\Lambda$ is given by

$$
\Lambda_{+}=\left\{\sum_{x \in X} c_{x} x \mid c_{x} \in \mathbb{Z}, \sum_{x \in X} c_{x} \equiv 0(\bmod 2)\right\} .
$$

Put $\Lambda_{-}=\Lambda \backslash \Lambda_{+}$. Then (20) implies

$$
\begin{array}{ll}
(\lambda, x) \equiv 0(\bmod 2) & \left(\lambda \in \Lambda_{+}, x \in X\right), \\
(\lambda, x) \equiv 1(\bmod 2) & \left(\lambda \in \Lambda_{-}, x \in X\right) .
\end{array}
$$

Consequently,

$$
\frac{1}{2} \Lambda_{+} \subset \Lambda^{*}
$$

3.2. The lattice $\Gamma$. In this subsection, we assume that there exists a tight spherical design $D=X \cup-X, X \cap-X=\varnothing$, and keep the notation of the preceding subsection. We consider the lattice $\Gamma$ defined by

$$
\Gamma=\frac{1}{\sqrt{2}} \Lambda_{+} .
$$

Note that $\Gamma$ is integral by (21). Our goal in this subsection is to study the structure of $\Gamma^{*} / \Gamma$. In certain cases, $\Gamma$ is shown to be even, so we can use the Milgram-Braun formula to derive some consequences.

Lemma 3.1. For each $x \in X, \frac{1}{\sqrt{2}} x+\Gamma$ is an element of order 2 in $\Gamma^{*} / \Gamma$.

Proof. For any $\lambda \in \Lambda_{+},\left(\frac{1}{\sqrt{2}} \lambda, \frac{1}{\sqrt{2}} x\right) \in \mathbb{Z}$ by (21). This implies $\frac{1}{\sqrt{2}} x \in\left(\frac{1}{\sqrt{2}} \Lambda_{+}\right)^{*}=\Gamma^{*}$. Since $x \notin \Lambda_{+}$, we have $\frac{1}{\sqrt{2}} x \notin \Gamma$. Also, since $2 x \in \Lambda_{+}$, we have $2 \cdot \frac{1}{\sqrt{2}} x \in \Gamma$. Therefore, $\frac{1}{\sqrt{2}} x+\Gamma \neq \Gamma$ and $2 \cdot\left(\frac{1}{\sqrt{2}} x+\Gamma\right)=\Gamma$.

In particular, we see that $\operatorname{det} \Gamma$ is even.

Let $D_{2}$ be the Sylow 2-subgroup of $\Lambda^{*} / \Lambda$, and let $T$ be the preimage of $D_{2}$ in $\Lambda^{*}$. Observe that

$$
T \cap T^{*}=\Lambda .
$$

Indeed, if $\alpha \in T \cap T^{*}$ and $\beta \in \Lambda^{*}$, then $|T: \Lambda| \alpha \in \Lambda$ and $\left|\Lambda^{*}: T\right| \beta \in T$, whence

$$
(|T: \Lambda| \alpha, \beta) \in \mathbb{Z} \quad \text { and } \quad\left(\alpha,\left|\Lambda^{*}: T\right| \beta\right) \in \mathbb{Z} .
$$

Since $|T: \Lambda|$ and $\left|\Lambda^{*}: T\right|$ are relatively prime, we obtain $(\alpha, \beta) \in \mathbb{Z}$. This proves that $\alpha \in \Lambda^{* *}=\Lambda$, whence $T \cap T^{*}=\Lambda$. An easy consequence of this is the following:

$$
T+T^{*}=\Lambda^{*} .
$$

Lemma 3.2. Suppose that there exists an odd integer $r$ such that $(\alpha, \alpha) \in \frac{1}{r} \mathbb{Z}$ for all $\alpha \in \Lambda^{*}$. Then $\Gamma^{*} / \Gamma \cong \mathbb{Z} / 2 \mathbb{Z} \oplus A$, where $A$ is an Abelian group satisfying $r A=0$.

Proof. By assumption, we have $(2 r \alpha, \beta) \in \mathbb{Z}$ for all $\alpha, \beta \in \Lambda^{*}$. This implies

$$
2 r\left(\Lambda^{*} / \Lambda\right)=0 .
$$

Thus, $D_{2}$ is elementary Abelian, so that $D_{2}$ has order at most $2^{n}$. Also, (27) implies that $(2 \alpha, \beta) \in \mathbb{Z}$ for all $\alpha, \beta \in T$. Therefore, $\sqrt{2} T$ is integral and, consequently, $\sqrt{2} T \subset$ $(\sqrt{2} T)^{*}=\frac{1}{\sqrt{2}} T^{*}$. Thus, $2 T \subset T^{*} \cap T=\Lambda$ by (25). We see that $D_{2}=T / \Lambda$ is a quotient of $T / 2 T \cong(\mathbb{Z} / 2 \mathbb{Z})^{n}$. Since $2 T$ is even and $\Lambda$ is odd, we have $2 T \neq \Lambda$. Thus, 
$D_{2}$ is an elementary Abelian group of order at most $2^{n-1}$. Since $\operatorname{det} \Gamma / 2$ is an integer by Lemma 3.1, and

$$
\frac{1}{2} \operatorname{det} \Gamma=\frac{1}{2} \cdot \frac{1}{\sqrt{2}^{2 n}} \operatorname{det} \Lambda_{+}=\frac{1}{2^{n+1}}\left|\Lambda: \Lambda_{+}\right|^{2} \operatorname{det} \Lambda=\frac{1}{2^{n-1}}\left|\Lambda^{*}: \Lambda\right|,
$$

we conclude that $\operatorname{det} \Gamma / 2$ is odd. Therefore, $\Gamma^{*} / \Gamma \cong \mathbb{Z} / 2 \mathbb{Z} \oplus A$ for some Abelian group $A$ of odd order. Now (27) implies $2 r A=0$, whence $r A=0$.

Lemma 3.3. If $\alpha \in \Lambda^{*}$, then

$$
\frac{m(m+1)(\alpha, \alpha)(3(\alpha, \alpha)-(2 m+1))}{6}
$$

is a nonnegative integer.

Proof. The difference of (18) and (17) is a nonnegative integer divisible by 12 , because $k^{4}-k^{2} \equiv 0(\bmod 12)$ for all $k \in \mathbb{Z}$.

Lemma 3.4. Suppose that $m(m+1)$ is not divisible by the square of an odd prime. Then

$$
(\alpha, \alpha) \in \frac{1}{3} \mathbb{Z} \quad \text { for all } \alpha \in T^{*} .
$$

If, moreover, $m \equiv 1(\bmod 3)$, then

$$
(\alpha, \alpha) \in \mathbb{Z} \quad \text { for all } \alpha \in T^{*} .
$$

Proof. Pick $\alpha \in T^{*}$ and put $(\alpha, \alpha)=\frac{p}{q}$, where $p, q \in \mathbb{Z}$ are relatively prime. Since $\left|T^{*}: \Lambda\right|$ is odd, we see that $q$ is odd. By Lemma 3.3, we have

$$
\frac{m(m+1) p(3 p-(2 m+1) q)}{3 q^{2}} \in \mathbb{Z}
$$

By assumption, it follows that $q$ is a power of 3 . Write $q=3^{t}$. Since $m(m+1) \not \equiv 0(\bmod 9)$ by assumption, we obtain

$$
p-(2 m+1) 3^{t-1} \equiv 0\left(\bmod 3^{2 t-1}\right)
$$

if $t \geq 1$. But this forces $t=1$ and $m \neq \equiv 1(\bmod 3)$. This completes the proof.

Lemma 3.5. Suppose that $m(m+1)=2^{r} a$, where $a$ is odd. Then

$$
2^{\left[\frac{r-1}{2}\right]}(\alpha, \alpha) \in \mathbb{Z} \quad \text { for all } \alpha \in T .
$$

Proof. Pick $\alpha \in T$. Since $|T: \Lambda|$ is a power of 2 , we can write $(\alpha, \alpha)=\frac{p}{2^{t}}$, where $p$ is odd and $t$ is a nonnegative integer. By Lemma 3.3 .

$$
\frac{2^{r} a p\left(3 p-(2 m+1) 2^{t}\right)}{2^{2 t+1}} \in \mathbb{Z}
$$

Thus, $2 t+1 \leq r$, or equivalently, $t \leq\left[\frac{r-1}{2}\right]$.

Lemma 3.6. Suppose that $m(m+1)$ is not divisible by the square of an odd prime, and that $m(m+1) \not \equiv 0(\bmod 8)$. Then

$$
\Gamma^{*} / \Gamma \cong \mathbb{Z} / 2 \mathbb{Z} \oplus(\mathbb{Z} / 3 \mathbb{Z})^{t}
$$

for some nonnegative integer $t$. If, moreover, $m \equiv 1(\bmod 3)$, then $t=0$.

Proof. By Lemmas 3.4 and 3.5 and relation (26), we have $(\alpha, \alpha) \in \frac{1}{3} \mathbb{Z}$ for all $\alpha \in \Lambda^{*}$. Then, by Lemma 3.2, we obtain (29). If, moreover, $m \equiv 1(\bmod 3)$, then Lemma 3.4 implies that $(\alpha, \alpha) \in \mathbb{Z}$ for all $\alpha \in \Lambda^{*}$, so that $t=0$ by Lemma 3.2

Lemma 3.7. If $m(m+1) \not \equiv 0(\bmod 8)$, then $(\lambda, \lambda) \equiv 0(\bmod 4)$ for all $\lambda \in \Lambda_{+}$, and $(\lambda, \lambda) \equiv 2 m+1(\bmod 4)$ for all $\lambda \in \Lambda_{-}$. In particular, $\Gamma$ is even. 
Proof. First, suppose $\lambda \in \Lambda_{+}$. By (23), $\frac{1}{2} \lambda \in \Lambda^{*}$, and in fact $\frac{1}{2} \lambda \in T$. Then Lemma 3.5 implies $\left(\frac{1}{2} \lambda, \frac{1}{2} \lambda\right) \in \mathbb{Z}$, whence $(\lambda, \lambda) \equiv 0(\bmod 4)$. Next, suppose $\lambda \in \Lambda_{-}$. Pick an arbitrary $x \in X$. Then $\lambda+x \in \Lambda_{+}$, so that $(\lambda+x, \lambda+x) \equiv 0(\bmod 4)$. By (21), we obtain $(\lambda, \lambda) \equiv(x, x) \equiv 2 m+1(\bmod 4)$.

Finally, we give some consequences of the Milgram-Braun formula.

Lemma 3.8. If $\Gamma$ is even and $m$ is even, then $\operatorname{det} \Gamma \neq 2$.

Proof. If $\operatorname{det} \Gamma=2$, then, by Lemma 3.1, we have $\Gamma^{*} / \Gamma=\left\{\Gamma, \frac{1}{\sqrt{2}} x+\Gamma\right\}$, where $x \in X$ is an arbitrary element. Since $\Gamma$ is even, we can apply the Milgram-Braun formula (10) to obtain $1+e^{\pi i(2 m+1) / 2}=\sqrt{2} e^{n \pi i / 4}$. Since $n \equiv-1(\bmod 8)$ and $m$ is even, we arrive at a contradiction.

Lemma 3.9. If $\operatorname{det} \Gamma=6$ and $\Gamma$ is even, then $m$ is even, and $(\alpha, \alpha) \equiv \frac{2}{3}(\bmod \mathbb{Z})$ whenever $\alpha+\Lambda$ is an element of order 3 in $\Lambda^{*} / \Lambda$.

Proof. Let $\alpha+\Lambda$ be an element of order 3 in $\Lambda^{*} / \Lambda$. Since $(3 \alpha, \alpha) \in \mathbb{Z}$, we can write $(\alpha, \alpha)=\frac{k}{3}$ for some positive integer $k$. Now $\sqrt{2} \alpha \in \sqrt{2} \Lambda^{*} \subset \sqrt{2} \Lambda_{+}^{*}=\left(\frac{1}{\sqrt{2}} \Lambda_{+}\right)^{*}=\Gamma^{*}$. If $\sqrt{2} \alpha \in \Gamma=\frac{1}{\sqrt{2}} \Lambda_{+} \subset \frac{1}{\sqrt{2}} \Lambda$, then $2 \alpha \in \Lambda$. Also, by assumption, $3 \alpha \in \Lambda$. Thus, $\alpha \in \Lambda$, a contradiction. Therefore, $\sqrt{2} \alpha \notin \Gamma$. Also, $3 \sqrt{2} \alpha \in \sqrt{2} \Lambda=\frac{1}{\sqrt{2}} 2 \Lambda \subset \frac{1}{\sqrt{2}} \Lambda_{+}=\Gamma$, which implies that $\sqrt{2} \alpha+\Gamma$ is an element of order 3 in $\Gamma^{*} / \Gamma$. Together with Lemma 3.1 this yields a set of representatives for $\Gamma^{*} / \Gamma$ :

$$
0, \frac{1}{\sqrt{2}} x, \sqrt{2} \alpha, \frac{1}{\sqrt{2}} x+\sqrt{2} \alpha, 2 \sqrt{2} \alpha, \frac{1}{\sqrt{2}} x+2 \sqrt{2} \alpha,
$$

where $x$ is an arbitrary element of $X$. Reduced modulo $2 \mathbb{Z}$, the norms of these elements are

$$
0, \frac{2 m+1}{2}, \frac{2 k}{3}, \frac{2 m+1}{2}+\frac{2 k}{3}, \frac{2 k}{3}, \frac{2 m+1}{2}+\frac{2 k}{3},
$$

respectively. Since $\Gamma$ is even, we can apply the Milgram-Braun formula (10) to get

$$
\left(1+e^{(2 m+1) \pi i / 2}\right)\left(1+2 e^{2 k \pi i / 3}\right)=\sqrt{6} e^{-\pi i / 4} .
$$

Since

and

$$
1+e^{(2 m+1) \pi i / 2}= \begin{cases}\sqrt{2} e^{\pi i / 4} & \text { if } m \text { is even } \\ \sqrt{2} e^{-\pi i / 4} & \text { if } m \text { is odd }\end{cases}
$$

$$
1+2 e^{2 k \pi i / 3}= \begin{cases}3 & \text { if } k \equiv 0(\bmod 3), \\ \sqrt{3} e^{\pi i / 2} & \text { if } k \equiv 1(\bmod 3), \\ \sqrt{3} e^{-\pi i / 2} & \text { if } k \equiv 2(\bmod 3),\end{cases}
$$

we conclude that $m$ is even and $k \equiv 2(\bmod 3)$.

3.3. The general case. The following theorem rules out the existence of tight spherical 5 -designs in $\mathbb{R}^{n}$ for some values of $n$. In the appendix it is shown that the number of such $n$ is infinite.

Theorem 3.1. Suppose that $m=2 k$ is even, $k \equiv 2(\bmod 3)$, and that both $k$ and $2 k+1$ are square-free. Then no tight spherical 5 -design exists in $\mathbb{R}^{n}$ with $n=(2 m+1)^{2}-2$.

Proof. If there exists a tight spherical 5-design in $\mathbb{R}^{n}$, then the lattice $\Gamma$ is even by Lemma 3.7. But Lemma 3.6 implies $\operatorname{det} \Gamma=2$, which contradicts Lemma 3.8 because $m$ is even. 
The above theorem rules out the existence of a tight spherical 5-design for $m=$ $4,10,22,28,34,46,52,58, \ldots$.

However, it does not rule out the existence of a tight spherical 5-design for the smallest open case, that is, the case of $m=3$. In order to deal with this case, we need to know elements of small norm in the lattice $\Lambda$ generated by a tight spherical 5 -design. In the remaining part of this subsection, we give some general results, assuming the existence of a tight spherical 5-design in $\mathbb{R}^{n}$ as in Subsections 3.1 and 3.2 .

Lemma 3.10. We have $\min \left(\Lambda^{*}\right) \geq(2 m+1) / 3$. For $\alpha \in \Lambda^{*},(\alpha, \alpha)=(2 m+1) / 3$ if and only if $(\alpha, x) \in\{0, \pm 1\}$ for all $x \in X$.

Proof. The assertions are easy consequences of Lemma 3.3.

Lemma 3.11. $\min \left(\Lambda_{+}\right) \geq 4(2 m+1) / 3$.

Proof. This is immediate from (23) and Lemma 3.10

Lemma 3.12. $\min (\Lambda)=2 m+1$.

Proof. By Lemma 3.11, it suffices to show that $(\lambda, \lambda) \geq 2 m+1$ for all $\lambda \in \Lambda_{-}$. By (6) and (22), we have $n_{0}(\lambda)=0$, so that $|X|=\sum_{k=1}^{\infty} n_{k}(\lambda)$. Thus, by (17), we have

$$
\begin{aligned}
(\lambda, \lambda) & =\frac{1}{2 m(m+1)(2 m+1)} \sum_{k=1}^{\infty} k^{2} n_{k}(\lambda) \\
& \geq \frac{|X|}{2 m(m+1)(2 m+1)}=2 m+1-\frac{2}{2 m+1} \\
& >2 m .
\end{aligned}
$$

Therefore, $(\lambda, \lambda) \geq 2 m+1$.

Lemma 3.13. Suppose $\alpha \in \Lambda^{*}$ and $(\alpha, \alpha)=\min (\alpha+\Lambda)$. Then $(\alpha, x) \in\{0, \pm 1, \ldots, \pm m\}$ for all $x \in X$.

Proof. For all $x \in X$ we have $(\alpha, \alpha) \leq(\alpha \pm x, \alpha \pm x)$. This implies $|(\alpha, x)| \leq(2 m+1) / 2$. Since $\alpha \in \Lambda^{*}$, we have $|(\alpha, x)| \in \mathbb{Z}$, and the result follows.

Lemma 3.14. Suppose $m \neq 1(\bmod 3)$. If $\lambda \in \Lambda_{+}$, then there exists an element $x \in$ $X \cup-X$ such that $(\lambda, x) \geq 4$.

Proof. Suppose $|(\lambda, x)|<4$ for all $x \in X$. Observe that $\frac{1}{2} \lambda \in \Lambda^{*}$ by (23). By (21), we find $n_{k}\left(\frac{1}{2} \lambda\right)=0$ except $k=0,1$. Then, by Lemma 3.10 we have

$$
\left(\frac{1}{2} \lambda, \frac{1}{2} \lambda\right)=\frac{2 m+1}{3}
$$

whence

$$
(\lambda, \lambda)=\frac{4(2 m+1)}{3} \notin \mathbb{Z} .
$$

This is a contradiction.

Lemma 3.15. Suppose $m \geq 3$. There is no element $\alpha \in \Lambda^{*}$ such that $(\alpha, x) \in\{ \pm 1, \pm 3\}$ for all $x \in X$.

Proof. Suppose $|(\alpha, x)|$ is 1 or 3 for all $x \in X$. Then (6), (17), and (18) read like this:

$$
\begin{aligned}
n_{1}(\alpha)+n_{3}(\alpha) & =2 m(m+1)\left(4 m^{2}+4 m-1\right), \\
n_{1}(\alpha)+9 n_{3}(\alpha) & =2 m(m+1)(2 m+1)(\alpha, \alpha), \\
n_{1}(\alpha)+81 n_{3}(\alpha) & =6 m(m+1)(\alpha, \alpha)^{2} .
\end{aligned}
$$


Eliminating $n_{1}(\alpha)$ and $n_{3}(\alpha)$, we find

$$
3(\alpha, \alpha)^{2}-10(2 m+1)(\alpha, \alpha)+9\left(4 m^{2}+4 m-1\right)=0 .
$$

This quadratic equation in $(\alpha, \alpha)$ has discriminant $52-8 m(m+1)<0$ if $m \geq 3$. This is a contradiction.

Let $\Lambda_{k}$ denote the set of elements of norm $k$ in $\Lambda$, where $k \in \mathbb{Z}$.

Lemma 3.16. If $m<7$, then $\Lambda_{2 m+1}=X \cup-X$.

Proof. Let $\lambda \in \Lambda_{2 m+1}$. Then, by Lemma 3.15, there exists an element $x \in X$ such that $|(\lambda, x)| \geq 5$. Replacing $x$ by $-x$ if necessary, we may assume that $(\lambda, x) \geq 5$ for some $x \in X \cup-X$. Then $(\lambda-x, \lambda-x) \in \Lambda_{+}$and

$$
\begin{aligned}
& (\lambda-x, \lambda-x)=2(2 m+1)-2(\lambda, x) \\
& \quad \leq 4 m-8=\frac{4(2 m+1)}{3}+\frac{4(m-7)}{3} \\
& \quad<\frac{4(2 m+1)}{3} .
\end{aligned}
$$

By Lemma 3.11 we conclude that $\lambda=x \in X \cup-X$.

3.4. The case of $n=47$. Now we restrict our attention to the case where $n=47$. Suppose that there exists a tight spherical 5 -design $X \cup-X$ in $\mathbb{R}^{47}$. We keep the notation of the preceding subsections.

Lemma 3.17. $\Lambda_{k}=\varnothing$ for $k=8,9,10,11,13,14$.

Proof. If $\lambda \in \Lambda_{+}$, then, by Lemma 3.11, we have $(\lambda, \lambda) \geq 28 / 3$. This implies $\Lambda_{8}=\varnothing$. Also, $\Lambda_{9}=\Lambda_{10}=\Lambda_{13}=\Lambda_{14}=\varnothing$ by Lemma 3.7. Let $\lambda \in \Lambda_{11}$. By Lemma 3.15 there exists an element $x \in X \cup-X$ such that $(\lambda, x) \geq 5$. Then $(\lambda-x, \lambda-x) \leq 8$. We have already shown that $\Lambda_{8}=\varnothing$, while $\lambda-x \in \Lambda_{+}$, so that $(\lambda-x, \lambda-x) \leq 6$. Therefore, $\lambda=x$ by Lemma 3.11, a contradiction.

Lemma 3.18. Every element of $\Lambda_{12}$ is uniquely expressible as the sum of two elements $x, y \in X \cup-X$ satisfying $(x, y)=-1$. In particular, $\left|\Lambda_{12}\right|=|X|(|X|-1)$.

Proof. Let $\lambda \in \Lambda_{12}$. By Lemma 3.14, there exists an element $x \in X \cup-X$ such that $(\lambda, x) \geq 4$. If $(\lambda, x)=4$, then $(\lambda-x, \lambda-x)=11$, contradicting Lemma 3.17. If $(\lambda, x) \geq 8$, then $(\lambda-x, \lambda-x) \leq 3$, a contradiction. Thus, we conclude that $(\lambda, x)=6$. Then $(\lambda-x, \lambda-x)=7$, whence $\lambda-x \in X \cup-X$ by Lemma 3.16

It remains to show the uniqueness of an expression $\lambda=x+y, x, y \in X$, with $(x, y)=$ -1 . Suppose $\lambda=x^{\prime}+y^{\prime}, x^{\prime}, y^{\prime} \in X \cup-X,\left(x^{\prime}, y^{\prime}\right)=-1$. If $x \neq x^{\prime}, y^{\prime}$, then $(x, \lambda)=$ $\left(x, x^{\prime}+y^{\prime}\right)= \pm 1 \pm 1 \leq 2$, while $(x, \lambda)=(x, x+y)=6$. This is a contradiction. Therefore, $x=x^{\prime}$ or $x=y^{\prime}$, and uniqueness follows.

Lemma 3.19. Suppose that $\alpha+\Lambda \in \Lambda^{*} / \Lambda$ has an odd order $r>1$. Then $\min (\alpha+\Lambda)=$ $28 / 3$.

Proof. Without loss of generality we may assume that $\min (\alpha+\Lambda)=(\alpha, \alpha)$. Then, by Lemma 3.13, we have

$$
(\alpha, x) \in\{0, \pm 1, \pm 2, \pm 3\} \quad \text { for all } x \in X .
$$

Since $r \alpha \in \Lambda=\Lambda_{+} \cup \Lambda_{-}$, first we consider the case where $r \alpha \in \Lambda_{-}$. Then $(r \alpha, x)=r(\alpha, x)$ is odd for all $x \in X$ by (22). Since $r$ is odd and $(\alpha, x) \in \mathbb{Z}$, we see that $(\alpha, x)$ is odd for all $x \in X$. Now (30) contradicts Lemma 3.15 Therefore, $r \alpha \in \Lambda_{+}$. Then $(r \alpha, x)$ is even, and $(\alpha, x)$ is also even. This implies $\left(\frac{1}{2} \alpha, x\right) \in\{0, \pm 1\}$ by (30), whence we obtain $\left(\frac{1}{2} \alpha, \frac{1}{2} \alpha\right)=\frac{7}{3}$ by Lemma 3.10 This proves that $\min (\alpha+\Lambda)=28 / 3$. 
Lemma 3.20. If $\alpha, \beta \in \Lambda^{*}$, then $24((\alpha, \alpha) \beta+2(\alpha, \beta) \alpha) \in \Lambda$.

Proof. By (19), we have

$$
24((\alpha, \beta) \gamma+(\alpha, \gamma) \beta+(\beta, \gamma) \alpha)=\sum_{x \in X}(\alpha, x)(\beta, x)(\gamma, x) x \in \Lambda .
$$

Putting $\gamma=\alpha$, we obtain the desired result.

Lemma 3.21. $\operatorname{det} \Gamma=2$.

Proof. By Lemma [3.6, we have $\Gamma^{*} / \Gamma \cong \mathbb{Z} / 2 \mathbb{Z} \oplus(\mathbb{Z} / 3 \mathbb{Z})^{t}$. First, we show that $t \leq 1$. By (28), it suffices to check that there are at most two elements of order 3 in $\Lambda^{*} / \Lambda$. Let $\alpha+\Lambda, \beta+\Lambda \in \Lambda^{*} / \Lambda$ be elements of order 3 . We want to show that $\beta+\Lambda= \pm(\alpha+\Lambda)$. By Lemma 3.19 we may assume

$$
(\alpha, \alpha)=(\beta, \beta)=\frac{28}{3} .
$$

If $\alpha+\beta \in \Lambda$, then $\beta+\Lambda=-\alpha+\Lambda$ and we are done. So, suppose $\alpha+\beta \notin \Lambda$. Then $\alpha+\beta+\Lambda$ is also an element of order 3. By Lemma 3.19.

$$
(\alpha+\beta, \alpha+\beta) \equiv \frac{28}{3}(\bmod \mathbb{Z}) .
$$

Now (31) and (32) imply

$$
(\alpha, \beta) \equiv \frac{1}{3}(\bmod \mathbb{Z})
$$

We have

$$
\beta+\Lambda=-24 \cdot \frac{28}{3} \beta+\Lambda=-24(\alpha, \alpha) \beta+\Lambda=48(\alpha, \beta) \alpha+\Lambda
$$

by Lemma 3.20. By (33), $\beta+\Lambda=16 \alpha+\Lambda=\alpha+\Lambda$. Therefore, we have shown that $t \leq 1$, whence $\operatorname{det} \Gamma=2$ or 6 . The latter case cannot occur. Indeed, by Lemma 3.7 $\Gamma$ is even, so that Lemma 3.9 yields a contradiction because $m=3$ is odd.

Starting with $\Gamma$, we can construct an even unimodular lattice $\tilde{\Gamma}$ of rank 48 from $\Gamma$ as follows. Consider the lattice $\sqrt{2} \mathbb{Z} \oplus \Gamma \subset \mathbb{R}^{48}$. We define $\tilde{\Gamma}$ by

$$
\tilde{\Gamma}=(\sqrt{2} \mathbb{Z} \oplus \Gamma) \cup\left(\left(\frac{1}{\sqrt{2}}, \frac{1}{\sqrt{2}} x_{0}\right)+(\sqrt{2} \mathbb{Z} \oplus \Gamma)\right),
$$

where $x_{0} \in X$. Note that the definition of $\tilde{\Gamma}$ is independent of the choice of $x_{0} \in X$ by Lemmas 3.1 and 3.6 Since $\Gamma$ is even by Lemma 3.7, $\sqrt{2} \mathbb{Z} \oplus \Gamma$ is also even. The norm of the element $\left(\frac{1}{\sqrt{2}}, \frac{1}{\sqrt{2}} x_{0}\right)$ is $\frac{1}{2}+\frac{7}{2}=4$. Therefore, we conclude that $\tilde{\Gamma}$ is even. Observe that $\operatorname{det}(\sqrt{2} \mathbb{Z} \oplus \Gamma)=\operatorname{det} \sqrt{2} \mathbb{Z} \operatorname{det} \Gamma=4$. Thus,

$$
\operatorname{det} \tilde{\Gamma}=\frac{1}{|\tilde{\Gamma}: \sqrt{2} \mathbb{Z} \oplus \Gamma|^{2}} \operatorname{det}(\sqrt{2} \mathbb{Z} \oplus \Gamma)=1 .
$$

Now we compute the theta series of $\tilde{\Gamma}$. In order to do this, we compute the number of elements of norm $2,4,6$ in $\tilde{\Gamma}$.

First, observe that $\Gamma^{*}=\frac{1}{\sqrt{2}} \Lambda$. Indeed, clearly we have $\frac{1}{\sqrt{2}} \Lambda \subset \Gamma^{*}$. But

$$
\left|\Gamma^{*}: \Gamma\right|=2=\left|\Lambda: \Lambda_{+}\right|=\left|\frac{1}{\sqrt{2}} \Lambda: \Gamma\right|,
$$

so that we must have $\frac{1}{\sqrt{2}} \Lambda=\Gamma^{*}$. Now, by Lemma 3.17, if $\alpha \in \Gamma^{*} \backslash \Gamma$ and $(\alpha, \alpha) \leq 6$, then $(\alpha, \alpha)=7 / 2$, whence $\sqrt{2} \alpha \in X \cup-X$ by Lemma 3.16. Thus, the only elements of $\tilde{\Gamma} \backslash(\sqrt{2} \mathbb{Z} \oplus \Gamma)$ having norm at most 6 are of the form $\left( \pm \frac{1}{\sqrt{2}}, \frac{1}{\sqrt{2}} x\right)$, where $x \in X \cup-X$. There are $4|X|$ such elements. 
The elements of norm at most 6 in $\sqrt{2} \mathbb{Z} \oplus \Gamma$ are

$$
( \pm \sqrt{2}, 0), \quad(0, \sqrt{2} \lambda) \quad\left(\lambda \in \Lambda_{12}\right) .
$$

By Lemma 3.18, there are $|X|(|X|-1)$ elements of norm 6 in $\sqrt{2} \mathbb{Z} \oplus \Gamma$. Therefore, the theta series $\vartheta_{\tilde{\Gamma}}(q)=\sum_{\lambda \in \tilde{\Gamma}} q^{(\lambda, \lambda) / 2}, q=e^{2 \pi i \tau}$, of $\tilde{\Gamma}$ is

$$
1+2 q+4|X| q^{2}+|X|(|X|-1) q^{3}+\cdots=1+2 q+4512 q^{2}+1271256 q^{3}+\cdots .
$$

It is known that the theta series of an even unimodular lattice of rank 48 is a linear combination of $\Delta^{2}, \Delta Q^{3}, Q^{6}$, where

$$
\begin{aligned}
& \Delta=q-24 q^{2}+252 q^{3}+\cdots \\
& Q=1+240 q+2160 q^{2}+6720 q^{3}+\cdots .
\end{aligned}
$$

Comparing the coefficients, we get a contradiction.

\section{§4. Tight 7-Designs}

4.1. Basic results. Suppose $D=X \cup-X \subset S^{n-1}(d) \subset \mathbb{R}^{n}$ is a tight spherical 7-design, where $X \cap-X=\varnothing$ and

$$
n=3 d^{2}-4 \quad(d \in \mathbb{N})
$$

Then

$$
\begin{aligned}
|X| & =\frac{n(n+1)(n+2)}{6}, \\
(x, y) & =0, \pm 1 \quad \text { for all } x, y \in X, x \neq y .
\end{aligned}
$$

Now the basic relations (7)-(9) can be written as

$$
\begin{aligned}
\sum_{k=1}^{\infty} k^{2} n_{k}(\alpha) & =\frac{1}{2}\left(3 d^{2}-2\right)\left(d^{2}-1\right) d(\alpha, \alpha), \\
\sum_{k=1}^{\infty} k^{4} n_{k}(\alpha) & =\frac{3}{2}\left(d^{2}-1\right) d^{2}(\alpha, \alpha)^{2}, \\
\sum_{k=1}^{\infty} k^{6} n_{k}(\alpha) & =\frac{5}{2}\left(d^{2}-1\right) d(\alpha, \alpha)^{3} .
\end{aligned}
$$

Let $\Lambda$ be the lattice generated by $X$. Then $\Lambda$ is even if and only if $d$ is even.

Lemma 4.1. If $\alpha \in \Lambda^{*}$ and $\alpha \neq 0$, then

and

$$
\frac{d\left(d^{2}-1\right)(\alpha, \alpha)\left(5(\alpha, \alpha)^{2}-15 d(\alpha, \alpha)+4\left(3 d^{2}-2\right)\right)}{48},
$$

$$
\frac{d\left(d^{2}-1\right)(\alpha, \alpha)\left(5(\alpha, \alpha)^{2}-\left(3 d^{2}-2\right)\right)}{120}
$$

are positive integers.

Proof. Observe that $k^{2}\left(k^{2}-1\right)\left(k^{2}-4\right) \equiv 0(\bmod 24), k^{2}\left(k^{2}-1\right) \equiv 0(\bmod 12)$, and $k^{2}\left(k^{4}-1\right) \equiv 0(\bmod 60)$. The assertions follow by appropriately combining the basic relations (37)-(39). 
Lemma 4.2. (i) Let $p \geq 5$ be a prime. If $\nu_{p}\left(d\left(d^{2}-1\right)\right)<3$, then $\nu_{p}((\alpha, \alpha)) \geq 0$ for all $\alpha \in \Lambda^{*}$.

(ii) If $\nu_{3}\left(d\left(d^{2}-1\right)\right)<4$, then $\nu_{3}((\alpha, \alpha)) \geq 0$ for all $\alpha \in \Lambda^{*}$.

(iii) If $\nu_{2}\left(d\left(d^{2}-1\right)\right)<7$, then $\nu_{2}((\alpha, \alpha)) \geq 0$ for all $\alpha \in \Lambda^{*}$. If $d$ is even but not divisible by 8 , then $\nu_{2}((\alpha, \alpha)) \geq 1$ for all $\alpha \in \Lambda^{*}$.

Proof. Taking $\alpha \in \Lambda^{*}$, we put $(\alpha, \alpha)=\frac{a}{b}$, where $a, b \in \mathbb{Z}$ are relatively prime. Since (40)- (42) are integers, we have

$$
\begin{aligned}
\nu_{p}\left(\frac{d\left(d^{2}-1\right) a^{2}\left(5 a^{2}-15 a b+4 b^{2}\left(3 d^{2}-2\right)\right)}{48 b^{3}}\right) & \geq 0, \\
\nu_{p}\left(\frac{d\left(d^{2}-1\right) a\left(3 a d-b\left(3 d^{2}-2\right)\right)}{24 b^{2}}\right) & \geq 0, \\
\nu_{p}\left(\frac{d\left(d^{2}-1\right) a\left(5 a^{2}-b^{2}\left(3 d^{2}-2\right)\right)}{120 b^{3}}\right) & \geq 0
\end{aligned}
$$

for every prime $p$. If $p>5$ and $\nu_{p}((\alpha, \alpha))<0$, then $p$ divides $b$, and from (45) we obtain $\nu_{p}\left(d\left(d^{2}-1\right)\right) \geq 3$.

If $\nu_{5}((\alpha, \alpha))<0$, then 5 divides $b$, and $\nu_{5}\left(5 a^{2}-b^{2}\left(3 d^{2}-2\right)\right)=1$. Thus, from (45) we obtain $\nu_{5}\left(d\left(d^{2}-1\right)\right) \geq 3$.

If $\nu_{3}((\alpha, \alpha))<0$, then 3 divides $b$, and from (45) we obtain $\nu_{3}\left(d\left(d^{2}-1\right)\right) \geq 4$.

If $\nu_{2}((\alpha, \alpha))<0$, then 2 divides $b$, and from (43) we obtain $\nu_{3}\left(d\left(d^{2}-1\right)\right) \geq 7$.

Finally, suppose that $d$ is even but not divisible by 8 ; we have already shown that $b$ is odd. By (44) and (45), we find

$$
\nu_{2}\left(\frac{d\left(d^{2}-1\right) a^{2}(5 a-3 b d)}{8}\right) \geq 0 .
$$

This forces $a$ to be even.

Theorem 4.1. Let $d>1$ be a positive integer, and suppose that $\nu_{2}(d)=2, \nu_{3}\left(d\left(d^{2}-1\right)\right)<$ 4 , and $\nu_{p}\left(d\left(d^{2}-1\right)\right)<3$ for every prime $p \geq 5$. Then a tight spherical 7 -design in dimension $n=3 d^{2}-4$ does not exist.

Proof. By Lemma 4.2, we see that $\Lambda^{*}$ is even, and, consequently, $\Lambda$ is an even unimodular lattice. But the dimension of $\Lambda$ is $n \equiv 4(\bmod 8)$, which is impossible.

In particular, now the first open case $d=4$ is settled by the above theorem. Also, the above theorem rules out the existence of a tight spherical 7-design for the cases where $d=12,20,28,36, \ldots$.

The next case of $d=5$ is due essentially to Martinet [1], since we can easily show that $\Lambda_{5}=D$, as follows.

Lemma 4.3. There is no element $\alpha \in \Lambda^{*}$ such that $(\alpha, x) \in\{0, \pm 1, \pm 2\}$ for all $x \in X$.

Proof. We have

$$
\begin{aligned}
n_{1}(\alpha)+4 n_{2}(\alpha) & =\frac{1}{2}\left(3 d^{2}-2\right)\left(d^{2}-1\right) d(\alpha, \alpha), \\
n_{1}(\alpha)+16 n_{2}(\alpha) & =\frac{3}{2}\left(d^{2}-1\right) d^{2}(\alpha, \alpha)^{2}, \\
n_{1}(\alpha)+64 n_{2}(\alpha) & =\frac{5}{2}\left(d^{2}-1\right) d(\alpha, \alpha)^{3} .
\end{aligned}
$$

Eliminating $n_{1}(\alpha), n_{2}(\alpha)$ from these equations, we find a quadratic equation in $(\alpha, \alpha)$ that has only imaginary solutions. This is a contradiction.

Theorem 4.2. A tight spherical 7-design in dimension $n=71$ does not exist. 
Proof. From Lemma 4.3 it follows easily that $\min \Lambda=5$ and $\Lambda_{5}=D$. Martinet [1] showed that $\Lambda_{5}$ cannot be a 7 -design. This is a contradiction.

4.2. The case of $n=104$. Now, we consider the next case: $d=6, n=104$. Here we give some properties of a possible lattice $\Lambda$. Since $d$ is even, $\Lambda$ is even. However, we have not been able to prove the nonexistence of a tight 7 -design in dimension $n=104$; the existence remains an interesting open problem.

Theorem 4.3. If a tight spherical 7-design $D$ in dimension 104 exists, then the lattice $\Lambda$ generated by $D$ has the following properties:

(i) $\Lambda$ is an even unimodular lattice.

(ii) $\Lambda$ has minimum 6 , and $\Lambda_{6}=D$.

(iii) $\Lambda_{8}=\varnothing$.

(iv) The theta series of $\Lambda$ is uniquely determined as

$$
1+385840 q^{3}+153139896000 q^{5}+1021417687180800 q^{6}+\cdots .
$$

(v) $\Lambda_{k}$ is a spherical 7-design for all $k=6,10,12,14, \ldots$.

Proof. (i) Lemma 4.2 shows that $\Lambda^{*}$ is even, whence $\Lambda$ is an even unimodular lattice.

(ii) We can show that there is no element $\alpha \in \Lambda^{*}$ such that $(\alpha, x) \in\{0, \pm 1, \pm 2, \pm 3\}$ for all $x \in X$. In fact, solving the basic relations for four unknowns $n_{k}(\alpha)(k=0,1,2,3)$, we find a unique solution containing a negative number. Then it follows easily that the minimum of $\Lambda$ is 6 . If $\alpha \in \Lambda_{6} \backslash D$, then, again, $(\alpha, x) \in\{0, \pm 1, \pm 2, \pm 3\}$ for all $x \in X$, which is impossible.

(iii) Suppose $\alpha \in \Lambda_{8}$. Since $\min \Lambda=6$, we see that $|(\alpha, x)| \leq 4$ for all $x \in X$. If $(\alpha, x)= \pm 4$ for some $x \in X$, then $\alpha \pm x \in \Lambda_{6}$. By (ii), $y:=\alpha \pm x \in D$. Then $|(x, y)|=2$, which is impossible. Therefore, $|(\alpha, x)|<4$ for all $x \in X$. Solving the basic equations, we obtain $n_{3}(\alpha)=56$. Put

$$
N_{3}=\{x \in D \mid(\alpha, x)=3\} .
$$

Then $\left|N_{3}\right|=56$. We claim that $\sum_{x \in N_{3}} x=21 \alpha$. Without loss of generality we may assume that $(\alpha, x) \geq 0$ for all $x \in X$. Then, by (4), $\sum_{x \in N_{3}} x$ is a scalar multiple of $\alpha$. The scalar is determined to be 21 , because $(\alpha, \alpha)=8$ and

$$
\sum_{x \in N_{3}}(\alpha, x)=3\left|N_{3}\right|=168=21 \cdot 8 .
$$

Pick an element $x_{0} \in N_{3}$. Then

$$
\begin{aligned}
63 & =\left(x_{0}, 21 \alpha\right)=\left(x_{0}, \sum_{x \in N_{3}} x\right)=6+\sum_{x \in N_{3} \backslash\left\{x_{0}\right\}}\left(x_{0}, x\right) \leq 6+\sum_{x \in N_{3} \backslash\left\{x_{0}\right\}}\left|\left(x_{0}, x\right)\right| \\
& \leq 6+55=61 .
\end{aligned}
$$

This is a contradiction.

(iv) Since the space of modular forms of weight 52 has dimension 5 , the theta series is uniquely determined by (ii), (iii).

(v) Let $f$ be a homogeneous harmonic polynomial of degree 2,4 , or 6 . Since $\Lambda_{6}$ is a spherical 6-design, by (ii) we have $\sum_{x \in \Lambda_{6}} f(x)=0$. The harmonic theta series

$$
\vartheta_{\Lambda, f}(q)=\sum_{x \in \Lambda} f(x) q^{(x, x) / 2}
$$

is a modular form of weight $52+\operatorname{deg} f$. Statements (ii) and (iii) imply that $\vartheta_{\Lambda, f}$ has no terms of degree less than 5 . The spaces of modular forms of weight 54,56 , and 58 all have dimension 5 , so that $\vartheta_{\Lambda, f}=0$. This implies that $\Lambda_{k}$ is a spherical 6-design, hence a 7 -design, for $k=10,12,14, \ldots$. 


\section{APPENDIX \\ BY Y.-F. S. PÉTERMANN}

Tight spherical 5-designs can possibly exist in dimension $n$ if $n \in \mathbb{N}_{5}:=\{n \in \mathbb{N} \mid$ $\left.n=(2 m+1)^{2}-2\right\}$, and tight spherical 7-designs can possibly exist in dimension $n$ if $n \in \mathbb{N}_{7}:=\left\{n \in \mathbb{N} \mid n=3 d^{2}-4\right\}$. In this Appendix we deduce from Theorem 3.19 that the set of integers for which no tight spherical 5-design exists in $\mathbb{R}^{n}$ has a positive density in $\mathbb{N}_{5}$, and from Theorem 4.3 we deduce that the set of integers for which no tight spherical 7-design exists in $\mathbb{R}^{n}$ has a positive density in $\mathbb{N}_{7}$. More precisely, if we denote by $N_{5}(x)$ (respectively, $N_{7}(x)$ ), the number of integers $n \leq x$ in $\mathbb{N}_{5}$ (respectively, in $\mathbb{N}_{7}$ ) for which there are no tight spherical 5-design (respectively, 7-design) in $\mathbb{R}^{n}$, then we have the following.

Corollary (to Theorems 3.10 and 4.3). We have ( $p$ denoting prime numbers)

$$
N_{5}(x) \geq \frac{1}{16} \prod_{p>3}\left(1-\frac{2}{p^{2}}\right) \sqrt{x}+O\left(x^{1 / 3} \log ^{3} x\right)=: K_{5} \sqrt{x}+O\left(x^{1 / 3} \log ^{3} x\right)
$$

and

$$
N_{7}(x) \geq \frac{1}{8 \sqrt{3}} \prod_{p \geq 3}\left(1-\frac{3}{p^{2}}\right) \sqrt{x}+O\left(x^{3 / 8+\epsilon}\right)=: K_{7} \sqrt{x}+O\left(x^{3 / 8+\epsilon}\right) .
$$

These lower bounds are obtained by computing the density of certain couples or triples of squarefree integers. This type of estimates is not new, and very similar problems have been considered since long ago (see [5, 13, 14]), the main difficulty being in general to obtain a good estimate for the remainder term. However, here, since the constants of the main terms on the right in (46) and (47) are very likely to be far from optimal, there seemed to be no point in this context to fight for better estimates of the remainder terms. So I chose the simplest proof I could have a hold on, and for this purpose I borrowed some ideas from D. R. Heath-Brown's elementary argument in [10] for a very similar problem. Note that even the much older elementary estimate obtained in 13 by Mirsky would yield (slightly) better estimates; on the other hand, Heath-Brown's sieve method in [10] would yield even better estimates.

Notation. In the sequel, $\mu$ denotes the Möbius function, $d$ the number of divisors function $d(n)=\sum_{n_{1} n_{2}=n} 1, d_{3}$ the function $d_{3}(n)=\sum_{n_{1} n_{2} n_{3}=n} 1$, and $\omega$ the number of distinct prime factors function $\omega(n)=\sum_{p \mid n} 1$. The symbol $(i ; j ; \ell ; m)=1$ will mean that any two of the integers $i, j, \ell, m$ have no prime factor in common.

I shall use the classical estimates

$$
\sum_{n \leq x} d(n) \sim x \log x, \quad \sum_{n \leq x} d_{3}(n) \sim \frac{1}{2} x \log ^{2} x, \quad d(x) \ll x^{\epsilon},
$$

and

$$
\sum_{n \leq x} 2^{\omega(n)} \sim A x \log x \text { (for some constant } A \text { ), as } x \rightarrow \infty .
$$

Proofs. We prove (46). Put $E(k)=1$ if $k$ is square-free and $E(k)=0$ otherwise. If we show that

$$
\sum_{k \leq x, k \equiv 2(\bmod 3)} E(k) E(2 k+1)=4 K_{5} x+O\left(x^{2 / 3} \log ^{3} x\right),
$$

then (46) will follow from Theorem 3.10. 
We have $E(k)=\sum_{j^{2} \mid k} \mu(j)$, whence

$$
\sum_{k \leq x, k \equiv 2(\bmod 3)} E(k) E(2 k+1)=\sum_{i, j} \mu(i) \mu(j) N(x, i, j)=\Sigma_{1}+\Sigma_{2},
$$

where $N(x, i, j)=\left|\left\{k \leq x\left|k \equiv 2(\bmod 3), i^{2}\right| k, j^{2} \mid 2 k+1\right\}\right|$; summation in $\Sigma_{1}$ is over $i j \leq y$, and that in $\Sigma_{2}$ is over the remaining $i, j$, where $y$ with $\sqrt{x}<y<x$ is to be defined later.

Since, by the Chinese Remainder Theorem, $N(x, i, j)=x /\left(3 i^{2} j^{2}\right)+O(1)$ if $(6 i, j)=$ $(3, i)=1$ and $N(x, i, j)=0$ otherwise, we have

$$
\begin{aligned}
\Sigma_{1} & =\frac{x}{3} \sum_{i j \leq y,(6 i, j)=(3, i)=1} \frac{\mu(i j)}{(i j)^{2}}+O\left(\sum_{m=i j \leq y} 1\right) \\
& =\frac{x}{3} \sum_{(6 i, j)=(3, i)=1} \frac{\mu(i j)}{(i j)^{2}}+O\left(x \sum_{m>y} d(m) m^{-2}\right)+O\left(\sum_{m \leq y} d(m)\right) \\
& =\frac{1}{3}\left(1-\frac{1}{2^{2}}\right) \prod_{p>3}\left(1-\frac{2}{p^{2}}\right) x+O\left(x y^{-1} \log y\right)+O(y \log y) \\
& =4 K_{5} x+O(y \log y) .
\end{aligned}
$$

Now if $N=N(I, J):=\mid\left\{(i, j, u, v), I<i \leq 2 I, J<j \leq 2 J, \mu(j) \neq 0,2 i^{2} u+1=\right.$ $\left.j^{2} v \leq x\right\} \mid$, then there is some $(I, J)$ with $I J \gg y$ and $I, J \ll x^{1 / 2}$ such that $N=N(I, J)$ is maximal (so that $\Sigma_{2} \ll N \log ^{2} x$ ). Any such $N$ satisfies

$$
N \ll \sum_{\substack{J<j \leq 2 J \\ \mu(j) \neq 0}} \sum_{u \leq x / I^{2}} \sum_{\substack{I<i \leq 2 I \\ 2 i^{2} u \equiv-1\left(\bmod j^{2}\right)}} 1 .
$$

If $p$ is any prime number, then the number of solutions $i$ modulo $p^{2}$ of $2 u i^{2} \equiv-1\left(\bmod p^{2}\right)$ is at most 2. Therefore, if $\mu(j) \neq 0$, then the number of solutions $i$ modulo $j^{2}$ of $2 u i^{2} \equiv-1\left(\bmod j^{2}\right)$ is at most $2^{\omega(j)}$, and

$$
\begin{aligned}
N & \ll \sum_{J<j \leq 2 J} \sum_{u \leq x / I^{2}}\left(1+I J^{-2}\right) 2^{\omega(j)}=x I^{-2}\left(1+I J^{-2}\right) \sum_{J<j \leq 2 J} 2^{\omega(j)} \\
& \ll x \log x\left(J I^{-2}+I^{-1} J^{-1}\right) \ll x y^{-1 / 2} \log x,
\end{aligned}
$$

where for the last estimate we consider the cases $I \geq J$ and $J \geq I$ separately. By setting $y=x^{2 / 3}$, we obtain (48).

We prove (47). The set of positive integers $d \leq 4 x$ with $\nu_{2}(d)=2, \nu_{3}\left(d\left(d^{2}-1\right)\right)<4$, and $\nu_{p}\left(d\left(d^{2}-1\right)\right)<3(p \geq 5)$ contains the set of positive integers $d=4 k \leq 4 x$ with $k$ odd and such that $k, 4 k-1$, and $4 k+1$ are all square-free. As before, the number of elements in the latter set is

$$
\sum_{k \leq x, k \equiv 1(\bmod 2)} E(k) E(4 k-1) E(4 k+1)=\sum_{i, j, \ell} \mu(i) \mu(j) \mu(\ell) N(x, i, j, \ell)=\Sigma_{1}+\Sigma_{2},
$$

where $N(x, i, j, \ell)=\left|\left\{k \leq x, k \equiv 1(\bmod 2), i^{2}\left|k, j^{2}\right| 4 k-1, \ell^{2} \mid 4 k+1\right\}\right| ;$ summation in $\Sigma_{1}$ is over $i j \ell \leq y$, and that in $\Sigma_{2}$ is over the remaining $i, j, \ell$, where $y$ with $\sqrt{x}<y<x$ is to be defined later. As before, from the Chinese Remainder Theorem it follows that $N(x, i, j, \ell)=x /\left(2(i j \ell)^{2}\right)+O(1)$ if $(i ; j ; \ell ; 2)=1$, and $N(x, i, j, \ell)=0$ otherwise. As 
before, we have

$$
\begin{aligned}
\Sigma_{1} & =\frac{x}{2} \sum_{i j \ell \leq y,(i ; j ; \ell ; 2)=1} \frac{\mu(i j \ell)}{(i j \ell)^{2}}+O\left(\sum_{m=i j \ell \leq y} 1\right) \\
& =\frac{x}{2} \sum_{(i ; j ; \ell ; 2)=1} \frac{\mu(i j \ell)}{(i j \ell)^{2}}+O\left(x \sum_{m>y} d_{3}(m) m^{-2}\right)+O\left(\sum_{m \leq y} d_{3}(m)\right) \\
& =4 \sqrt{3} K_{7} x+O\left(y \log ^{2} y\right),
\end{aligned}
$$

and if $N=N(I, J, L):=\mid\{(i, j, \ell, u, v, w), I<i \leq 2 I, J<j \leq 2 J, \mu(j) \neq 0, L<\ell \leq$ $\left.2 L, 4 i^{2} u=j^{2} v+1=\ell^{2} w-1 \leq x\right\} \mid$, then there is some $(I, J, L)$ with $I J L \gg y$ and $I, J, L \ll x^{1 / 2}$ such that $N=N(I, J, L)$ is maximal, so that $\Sigma_{2} \ll N \log ^{3} x$.

Now for each $j$ with $J<j \leq 2 J$ and $\mu(j) \neq 0$, the number of solutions $i$ modulo $j^{2}$ of $4 u i^{2} \equiv 1\left(\bmod j^{2}\right)$ is at most $2^{\omega(j)}$. Since for every such solution $i$ the number of $\ell$ with $L<\ell \leq 2 L$ for which $i$ is also a solution of $4 u i^{2} \equiv-1\left(\bmod \ell^{2}\right)$ is at most $d\left(4 u i^{2}+1\right)$, that is, since $4 u i^{2} \leq x$, at most $x^{\epsilon}$, we have

$$
N_{I, L}:=\sum_{\substack{i, \ell \\ I<i \leq 2 I \\ L<\ell<2 L \\ 4 u i^{2} \equiv 1\left(\bmod j^{2}\right) \\ 4 u i^{2} \equiv-1\left(\bmod \ell^{2}\right)}} 1 \ll x^{\epsilon} 2^{\omega(j)}\left(1+I J^{-2}\right) .
$$

Thus, as before, we obtain

$$
N \ll \sum_{\substack{J<j \leq 2 J \\ \mu(j) \neq 0}} \sum_{u \leq x / I^{2}} N_{I, L} \ll x^{1+\epsilon}\left(J I^{-2}+I^{-1} J^{-1}\right) .
$$

Now by exchanging the roles of $I, J, L$, we also compute $N_{I, J}, N_{J, I}, N_{J, L}, N_{L, J}, N_{L, I}$; for instance,

$$
N_{J, I}:=\sum_{\substack{j, i \\ J<j \leq 2 J \\ I<i \leq 2 I \\ v j^{2} \equiv-2\left(\bmod \ell^{2}\right) \\ v j^{2} \equiv-1\left(\bmod i^{2}\right)}} 1 \ll x^{\epsilon} 2^{\omega(\ell)}\left(1+J L^{-2}\right),
$$

whence

$$
N \ll \sum_{\substack{L<\ell \leq 2 L \\ \mu(\ell) \neq 0}} \sum_{v \leq x / J^{2}} N_{J, I} \ll x^{1+\epsilon}\left(L J^{-2}+J^{-1} L^{-1}\right) .
$$

Hence,

$$
\begin{array}{r}
N \ll x^{1+\epsilon} \min \left(J I^{-2}+I^{-1} J^{-1}, I J^{-2}+J^{-1} I^{-1}, J L^{-2}+L^{-1} J^{-1},\right. \\
\left.L J^{-2}+J^{-1} L^{-1}, I L^{-2}+L^{-1} I^{-1}, L I^{-2}+I^{-1} L^{-1}\right) \\
\ll x^{1+\epsilon} \min \left(I^{-1}, J^{-1}, L^{-1}\right) \ll x^{1+\epsilon} y^{-1 / 3} \ll x^{3 / 4+\epsilon}
\end{array}
$$

if we set $y=x^{3 / 4}$. This yields (47).

\section{REFERENCES}

[1] E. Bannai and E. Bannai, Algebraic combinatorics on spheres, Springer-Verlag, Tokyo, 1999. (Japanese)

[2] E. Bannai and R. Damerell, Tight spherical designs. I, J. Math. Soc. Japan 31 (1979), 199-207. MR0519045 (80b:05014)

[3] , Tight spherical designs. II, J. London Math. Soc. (2) 21 (1980), 13-30. MR0576179 (81g:05024) 
[4] E. Bannai and N. J. A. Sloane, Uniqueness of certain spherical codes, Canad. J. Math. 33 (1981), 437-449. MR0617634 (83a:94020)

[5] L. Carlitz, On a problem of additive arithmetic. II, Quart. J. Math. Oxford Ser. 3 (1932), 273-290.

[6] J. H. Conway and N. J. A. Sloane, Sphere packing, lattices and groups, 3rd ed., Grundlehren Math. Wiss., vol. 290, Springer-Verlag, New York, 1999. MR1662447 (2000b:11077)

[7] P. Delsarte, J.-M. Goethals, and J. J. Seidel, Spherical codes and designs, Geom. Dedicata 6 (1977), 363-388. MR0485471 (58:5302)

[8] J.-M. Goethals and J. J. Seidel, Spherical designs, Relations between Combinatorics and other Parts of Mathematics (Proc. Sympos. Pure Math., Ohio State Univ., Columbus, Ohio, 1978), Proc. Sympos. Pure Math., vol. 34, Amer. Math. Soc., Providence, RI, 1979, pp. 255-272. MR0525330 (82h:05014)

[9] — The regular two-graph on 276 vertices, Discrete Math. 12 (1975), 143-158. MR0384597 $(52: 5471)$

[10] D. R. Heath-Brown, The square sieve and consecutive square-free numbers, Math. Ann. 266 (1984), 251-259. MR0730168 (85h:11050)

[11] J. Martinet, Sur certains designs sphériques liés à des réseaux entiers, Réseaux Euclidiens, Designs Sphériques et Formes Modulaires, Monogr. Enseign. Math., vol. 37, Enseign. Math., Gèneve, 2001, pp. 135-146. MF 1878748 (2002k:11107)

[12] J. Milnor and D. Husemoller, Symmetric bilinear forms, Ergeb. Math. Grenzgeb., vol. 73, SpringerVerlag, New York-Heidelberg, 1973. MF 0506372 (58:22129)

[13] L. Mirsky, On the frequency of pairs of square-free numbers with a given difference, Bull. Amer. Math. Soc. 55 (1949), 936-939. MF 0031507 (11:161e)

[14] _ Arithmetical pattern problems relating to divisibility by r-th powers, Proc. London Math. Soc. (2) 50 (1949), 497-508. MR0028334(10:431d)

[15] G. Nebe and B. Venkov, The strongly perfect lattices of dimension 10, J. Théor. Nombres Bordeaux 12 (2000), 503-518. MR1823200 (2002f:11081)

[16] B. Venkov, Réseaux et designs sphériques, Réseaux Euclidiens, Designs Sphériques et Formes Modulaires, Monogr. Enseign. Math., vol. 37, Enseign. Math., Gèneve, 2001, pp. 10-86. MR1878745 (2002m:11061)

Graduate School of Mathematics, Kyushu University, Hakozaki 6-10-1, Higashi-ku, FukuoKA $812-8581$, JAPAN

E-mail address: bannai@math.kyushu-u.ac.jp

Graduate School of Information Sciences, Tohoku University, Aramaki-Aza-Aoba 09 AobaKU, SENDAi 980-8579, JAPAN

E-mail address: munemasa@math.is.tohoku.ac.jp

St. Petersburg Branch, Steklov Mathematical Institute, Russian Academy of Sciences, Fontanka 27, St. Petersburg 191023, Russia

E-mail address: bbvenkov@yahoo.com

Section de Mathématiques, Université de Gèneve, Rue du Lievre 2-4, C.P. 240, 1211 Gèneve 24, SuISSE

E-mail address: petermann@math.unige.ch

Received 3/SEP/2003

Originally published in English 